\title{
La discriminación en el acceso a la información en la legislación española de discapacidad
}

\section{Discrimination in access to information in Spanish disability legislation}

Miguel Vieito-Villar, Universidad de Santiago de Compostela - miguel.vieito@usc.es

\section{Abstract}

It is our objective to show, in this paper, an overview of the Spanish regime of discrimination, in the right to information. In this sense, if the sender interposes obstacles or barriers to access, based on no rational criteria, we will be in a case of our study, a case of discrimination. We will stop on the legal provisions established for the benefit of disabled people, for the right to information,. In this regard, we also observe the legal regime established for cases where there has been a discriminatory act. It might seem that, in the year 2014 and in a digital era of free access to contents, information flies to those in need, without restrictions of any kind. Well, this thought, that may be true for certain subjects, it is not for many others as well the format of the information, but its contents are not set as accessible.

\section{Keywords}

Discrimination, disability, access to information, sign language.

\section{Resumen}

Es nuestra pretensión hacer ver, con el presente trabajo, una visión general del régimen español de vulneración del derecho de información por vía de discriminación. Es decir, si el medio emisor, o una persona intermediaria entre el emisor y el receptor de la información, interpone trabas u obstáculos al acceso, basados en criterios no racionales, estaremos en un caso objeto de nuestro estudio, es decir, un caso de discriminación. Observaremos las previsiones legales que, para el derecho a informarse, se establecen en beneficio de personas con discapacidad. En este sentido, observaremos también el régimen sancionador establecido para aquellos casos en que se haya producido un acto discriminador. Pudiera parecer que, en pleno año 2014 y en plena era digital de acceso libre a contenidos, la información vuela a quien la necesita, sin cortapisas de ningún tipo; basta pues con desear una información para poder obtenerla de manera casi instantánea. Pues bien, este pensamiento, que puede ser cierto para determinados sujetos, no lo es para muchos otros pues bien el formato de la información, bien su contenido, no se establecen como accesibles.

\section{Palabras clave}

Discriminación, discapacidad, acceso a información, lengua de signos. 


\section{Introducción}

Como todo artículo de trasfondo jurídico, nuestro punto de partida debe ser la Constitución de 1978. En este sentido, el Derecho a «comunicar y recibir información veraz por cualquier medio de difusión» se encuentra recogido en el artículo 20, es decir, en el bloque de artículos que se corresponde con los Derechos Fundamentales y Libertades Públicas. Dicho de otro modo, el Legislador Constituyente quiso para España, en el momento de redacción del Texto, que el Derecho a la información tuviese el mayor grado de protección que el Ordenamiento Jurídico español reconoce ${ }^{1}$. Con esto, se ha equiparado en configuración y naturaleza al Derecho a la vida, a la integridad física, a la libertad, a la educación...

Pero dando un paso más, no solo se trata de un Derecho Fundamental sino que, en una interpretación extensiva, opinamos que la información se erige realmente como uno de los principios fundamentales del Estado Constitucional, vinculado al Título Preliminar, esto es, a aquellas ideas inspiradoras que hablan no ya del sistema que la Constitución diseña, sino que hablan de lo que la Constitución es. De este modo, no se nos antoja como posible un Estado que «propugna como valores superiores de su ordenamiento jurídico la libertad, la justicia, la igualdad y el pluralismo político», sin que exista un flujo de información en los ciudadanos.

Es por esto que la información ha preocupado y preocupa enormemente al Derecho en nuestro país. Tenemos una buena cantidad de normas que afectan a aquella, agrupadas por sectores. A modo de ejemplo:

- $\quad$ Publicidad: Ley $34 / 1988$, de 11 de noviembre, General de Publicidad.

- Información Pública:Ley 19/2013, de 9 de diciembre, de transparencia, acceso a la información pública y buen gobierno.

- Límites en relación a la esfera íntima de otros: Ley Orgánica 1/1982, de 5 de mayo, sobre protección civil del derecho al honor, a la intimidad personal y familiar y a la propia imagen.

- Información y privacidad: Ley Orgánica 15/1999, de 13 de diciembre, de Protección de Datos de Carácter Personal.

- Información en Red: Ley 34/2002, de 11 de julio, de servicios de la sociedad de la información y de comercio electrónico.

Y la lista podría incrementarse enormemente por lo que podemos ver que, según el ámbito concreto al que nos refiramos, el régimen legal de la información puede variar. Así mismo, y ante tal cantidad de normas, en ocasiones puede resultar confuso discernir qué Ley es aplicable a qué caso concreto. Por esto, y sin que la extensión ni la pretensión de este trabajo nos permitan un análisis pormenorizado de todas ellas, nos referiremos en exclusiva a un campo concreto: la discriminación en el acceso a la información por razón de discapacidad.

\subsection{Definiciones}

Una de las primeras dificultades a la hora de hablar de discapacidad e información es, desde luego, qué entender por una y otra. Se trata de conceptos muy utilizados pero con una carga técnica importante que es necesario clarificar. Además, se trata de ideas que no siempre han tenido la misma caracterización y que, al menos en el campo de la discapacidad, han sido fruto de una conquista histórica.

Comenzando por la discapacidad indicaremos, como hemos apuntado, que no siempre ha sido el término elegido. En el pasado se han utilizado otros, marcados por un profundo e innegable peso peyorativo: impediso, tullido, incapaz, subnormal, aquel que no puede gobernarse por sí mismo...Si bien hoy día algunos de estos términos subsisten², incluso en nuestra legislación, sí es cierto que podemos hablar de una definición de consenso, alcanzada tras décadas de reformulaciones. Así, hoy entendemos, a nivel legal, que el término incluye a las personas:

«que tengan deficiencias físicas, mentales, intelectuales o sensoriales a largo plazo que, al interactuar con diversas barreras, puedan impedir su participación plena y efectiva en la sociedad, en igualdad de condiciones con las demás»

Se trata, como vemos, de una definición psico-social, es decir, aquella que define la discapacidad no solo en base a una patología (definición médica) sino en base a una serie de barreras sociales. De este modo, no solo la patología define al individuo sino que es la sociedad, al no remover sus barreras, la que define en buena parte una situación de discapacidad. Así mismo se trata de un término inclusivo, por cuanto todos nos encontramos en algún momento de nuestras vidas, con barreras que nos impiden una participación social y pública plenas.

La definición que acabamos de ver nace de un contexto internacional para, posteriormente, adaptarse a nuestro Derecho interno. En este sentido, es el Convenio de Nueva York, sobre derechos de las personas con discapacidad, es el que la emplea por primera vez. Por su

\footnotetext{
${ }^{1}$ Los Derechos Fundamentales en nuestro país: deben desarrollarse por Ley Orgánica (una norma con un sistema reforzado de mayorías en la aprobación y modificación), son directamente exigibles en Tribunales aun sin Ley que los desarrolle y, además, son susceptibles de recurso de amparo ante el Tribunal Constitucional.

${ }^{2}$ El Código Civil y la Ley de Enjuiciamiento Civil siguen hablando, pese a las críticas recibidas, de procedimiento de incapacitación, para referirse a aquel en el que se decide el grado de capacidad de un sujeto y, de ser el caso, los mecanismos de suplemento.
} 
influencia, toda la legislación española en la materia ha introducido la misma definición (por obra de la Ley de adaptación a la Convención, de 2011).

Para referimos al término información, por contra, no poseemos referencia legal alguna. En este sentido, ninguna de las leyes sobre derechos de las personas con discapacidad contiene definiciones sobre qué entender por información a efectos de su regulación. En este sentido, y en virtud de principios inspiradores que veremos a continuación, vamos a entender información en el sentido más amplio posible. Proponemos la siguiente definición, unicamente a efectos del presente trabajo: aquellos datos o elementos de conocimiento a través de los cuales el individuo es capaz de formar opiniones, razonamientos o su propia personalidad. Incluiremos, por tanto, desde datos académicos a cuestiones culturales y artísticas, opiniones políticas y cualesquiera otras de naturaleza similar.

\section{Regimen general de la discapacidad}

La regulación española de discapacidad es, en la actualidad, numerosa y confusa. De este modo, y por motivaciones diversas, los diferentes Gobiernos de la Nación se han ocupado de publicar normas, de contenido diverso y no siempre coordinadas entre sí, en esta materia. El origen de las más modernas leyes sobre discapacidad en nuestro país es la Convención de Nueva York, de la que ya hemos hablado. Con sus trabajos previos, su firma por el Estado español y su posterior entrada en vigor, se desencadenan en España cambios normativos y creación legislativa ciertamente destacables. Con esto, veremos a continuación las previsiones del antedicho Convenio en materia de personas con discapacidad e información.

\subsection{El Convenio de Nueva York}

Sin que sea nuestra intención detenernos en cuestiones introductorias sí queremos hacer ver la importancia capital de este texto internacional. En primer lugar, y debido a su contenido, podemos decir que se trata del Convenio sobre Derechos Humanos más importante del siglo XXI, y de los últimos años del XX. Esto es así pues los diferentes Estados que lo han ratificado se han sometido a un régimen de compromisos en materia de discapacidad con un alcance que no se había visto con anterioridad. Se trata de obligaciones en materia legislativa y administrativa que alcanzan a todos los niveles de la organización territorial estatal: central, autonómico y local. En segundo lugar el Convenio es único por su elevado nivel de concreción y su amplio espectro de aplicación. En este sentido, el Texto se ocupa de materias que abarcan desde la Sanidad, a la Información, pasando por Educación, Accesibilidad, especial atención a la niñas y mujeres con discapacidad y, sobre todo, asentando todo el conjunto sobre los principios de autonomía, libertad en la toma de decisiones, concepción dinámica de la discapacidad y empoderamiento de las personas con discapacidad. En tercer lugar el Convenio tiene un impacto a tener en cuenta por el número de países que lo han ratificado. En la actualidad un total de 151 países ha ratificado el texto del Convenio y un total de 159 lo ha firmado ; esto es, prácticamente tres cuartas partes de los Estados ha ratificado, con lo que el régimen de protección tiende a universalizarse. En cuarto lugar, Nueva York supone un instrumento de defensa de Derechos Humanos muy potente por la existencia de su Protocolo Adicional. Se trata de un Anexo al texto principal y que faculta a los individuos de los Estados a presentar, directamente a Naciones Unidas, sus quejas o denuncias por incumplimiento de los compromisos del cuerpo del Convenio. Si bien su extensión es más limitada, 85 países se han adherido al Protocolo, el número de ratificaciones crece poco a poco.

Centrándonos ya en el texto en sí, debemos comenzar por su justificación, su finalidad y sus principios rectores, en la medida en que resulten aplicables al Derecho a la información. En este sentido, debemos indicar que esta es una cuestión subyacente a todo el Convenio, pero que aflora explícitamente en varios puntos del mismo. Así, se encuentra implícita en sus Considerandos h y o:

- «h) Reconociendo también que la discriminación contra cualquier persona por razón de su discapacidad constituye una vulneración de la dignidad y el valor inherentes del ser humano

- $\quad$ o) Considerando que las personas con discapacidad deben tener la oportunidad de participar activamente en los procesos de adopción de decisiones sobre políticas y programas, incluidos los que les afectan directamente»

Así mismo, podemos establecer una relación obvia entre el Derecho a la información y los principios rectores del Convenio, en concreto los de: no discriminación, igualdad de oportunidades y participación e inclusión plenas y efectivas en la sociedad.

De modo claro y explícito, podemos ver reflejado el Derecho a información en el considerando v del Convenio, por cuanto indica:

- «v) Reconociendo la importancia de la accesibilidad al entorno físico, social, económico y cultural, a la salud y la educación y a la información y las comunicaciones, para que las personas con discapacidad puedan gozar plenamente de todos los derechos humanos y las libertades fundamentales»

Además, vemos la importancia del respeto de este Derecho en el principio básico de accesibilidad universal.

Si bien pueda parecer que estos principios y Considerandos son cuestiones menores, sin trascendencia jurídica, esta conclusión no sería, en absoluto, acertada. Estos realizan una función esencial en el sistema del Convenio y, además, en los sistemas nacionales. En cuanto a la primera, son un criterio inspirador e interpretador; con esto, deben emplearse como referencia a la hora de dilucidar cuestiones jurídicas confusas y en las que no sepamos si restringir o ampliar la esfera de actuación. Si, viendo este razonamiento en un ejemplo, dudamos si una persona con discapacidad debe tener acceso a subtítulos en un cine pudiéramos dudar pues el Convenio no se refiere específicamente a esta cuestión. Podríamos interpretar restrictivamente el Texto y concluir que no es aplicable a esto pero erraríamos pues los principios de participación plena y de accesibilidad universal deben hacernos interpretar este acceso a información y bienes culturales del modo más amplio posible, en una tendencia maximizadora. Vemos también, por los Considerandos, que fue esta en concreto la voluntad de los redactores del Texto. Como hemos dicho estas proclamaciones introductorias tienen también importancia a nivel nacional 
por cuanto fijan, para los Estados que han ratificado, unos mínimos infranqueables y que no pueden ser eliminados. Así, toda Nación que quiera aplicar en su territorio el Convenio, tendrá los mismos elementos estructurales; con la consecuente seguridad jurídica que de esto se deriva.

Llegando ya especificamente al Derecho a la información, comenzaremos indicando que es una materia regulada específicamente en el articulado, en concreto en el artículo 21, rotulado «Libertad de expresión y de opinión y acceso a la información»:

- «Los Estados Partes adoptarán todas las medidas pertinentes para que las personas con discapacidad puedan ejercer el derecho a la libertad de expresión y opinión, incluida la libertad de recabar, recibir y facilitar información e ideas en igualdad de condiciones con las demás y mediante cualquier forma de comunicación que elijan con arreglo a la definición del artículo 2 de la presente Convención, entre ellas:

Facilitar a las personas con discapacidad información dirigida al público en general, de manera oportuna y sin costo adicional, en formatos accesibles y con las tecnologías adecuadas a los diferentes tipos de discapacidad

Aceptar y facilitar la utilización de la lengua de señas, el Braille, los modos, medios, y formatos aumentativos y alternativos de comunicación y todos los demás modos, medios y formatos de comunicación accesibles que elijan las personas con discapacidad en sus relaciones oficiales

Alentar a las entidades privadas que presten servicios al público en general, incluso mediante Internet, a que proporcionen información y servicios en formatos que las personas con discapacidad puedan utilizar y a los que tengan acceso

Alentar a los medios de comunicación, incluidos los que suministran información a través de Internet, a que hagan que sus servicios sean accesibles para las personas con discapacidad

Reconocer y promover la utilización de lenguas de señas»

Lo primero que puede llamarnos la atención es lo ambicioso de las proclamaciones del artículo. Por él, los Estados se comprometen, en los puntos a) y b), a una serie de acciones directas; no obstante, en los siguientes, se comprometen incluso a influir en entes privados y en el modo en que estos prestan sus servicios. Se trata de una concreción de los principios que hemos visto y del propio espíritu del Convenio: los Estados deben hacer todo lo posible por remover la discriminación de sus territorios, incluso de las personas jurídicas. También destaca la universalidad en el acceso: las personas con discapacidad deben tener acceso a toda fuente de información, incluidas las electrónicas. Por último vemos también notas de una visión dinámica y particularizada de la discapacidad. En este sentido, los formatos disponibles deben estarlo en función de cada tipo de discapacidad.

¿A qué tiene derecho una persona con discapacidad en el Convenio? A «recabar, recibir y facilitar información», es decir, no solo a convertirse en sujeto pasivo de la emisión de información por parte de otros sino también a tener disponibles los medios suficientes para poder convertirse en agente activo de emisión de información y opiniones. Esto no implica, por supuesto, que una persona con discapacidad pueda exigir de todos los entes privados que emiten información una adaptación. No todos pueden afrontar la adaptación de formatos. Lo que si puede exigirse es, por un lado, que el Estado haga todos los esfuerzos posibles para que una fuente determinada de información esté accesible a todos; por otro, puede exigir una persona con discapacidad que la limitación de acceso no se produzca de modo discriminatorio, esto es, que la razón por la que un medio niegue la adaptación sea la propia discapacidad. Con esto, si son razones económicas, por ejemplo, las que impiden prestar el servicio en formatos accesibles, y estas razones se fundamentan y son reales, no habrá problema de legalidad.

El Convenio hace mención especial a dos modos de emitir y recibir información: el Braille y la Lengua de Signos. En cuanto a estos, los Estados se han obligado a facilitar información accesible al público en estos dos formatos. Además, deben autorizar la comunicación por estos medios a las personas con discapacidad en sus relaciones con la Administración. Es una cuestión de gran importancia por cuanto, pese al compromiso del Estado español, esta situación de normalización de la Lengua de Signos y el Braille no se ha producido en absoluto.

\section{Discriminación en el acceso a información}

Una vez visto el marco internacional para el Derecho a información, corresponde ahora examinar el sistema nacional. Como ya hemos dicho este bebe directamente de Nueva York y tiene como pilares esenciales sus pronunciamientos. Además de esto, el sistema nacional suplirá una carencia importante del sistema internacional: el sistema de sanciones e infracciones. Más allá de las posibilidades de denuncia que proporciona el Protocolo Adicional, el Convenio no establece una lista de infracciones y sanciones, convirtiéndose, en parte, en una norma imperfecta.

Veremos en este apartado dos disposiciones. En primer lugar, y como enlace con la parte final del apartado dedicado al Convenio, veremos la Ley que reconoce la Lengua de Signos española y la Lengua de Signos Catalana. Tras esto, analizaremos, a efectos de Derecho de información, la que es hoy la Ley más importante en materia de discapacidad: el Texto Refundido de la Ley General de derechos de las personas con discapacidad de 2013.

\subsection{La Ley $27 / 2007$ de reconocimiento de la Lengua de Signos}

El artículo 21.e) del Convenio, que hemos reproducido, exigía del Gobierno español el reconocimiento de la Lengua de Signos. Pues bien, en cumplimiento de tal mandato se publica en nuestro país la Ley 27/2007, de 23 de octubre, por la que se reconocen las lenguas de signos españolas y se regulan los medios de apoyo a la comunicación oral de las personas sordas, con discapacidad auditiva y sordociegas. Lo primero que debemos indicar es que con esta Ley no se establece un estatuto de oficialidad para la Lengua de Signos en nuestro país. Se trata de una cuestión sobre la que existe cierto nivel de confusión pero en ningún momento la Ley pretende dar a la Lengua de Signos, española o catalana, el mismo nivel jurídico que a las lenguas cooficiales del Estado. De echo, en su articulado, solo 
encontramos el término «oficial» u «oficialidad» para referirse, precisamente, a la lengua oral. Lo que se pretende con esta norma es reconocer la existencia de la lengua de signos, y desprender de este reconocimiento una serie de efectos jurídicos. Observando el resto del Ordenamiento Jurídico español tampoco encontramos disposiciones que completen un régimen de oficialidad. No existe pues para la Lengua de Signos ni regulación en Ley Orgánica, ni reflejo en Estatutos de Autonomía, ni protección por reconocimiento directo en Derechos Fundamentales, ni posibilidad clara e indubitada de emplearse en las relaciones con la Administración.

Se trata de una Ley limitada también en su extensión, debido a su estatuto de norma básica. ¿Qué quiere esto decir? Que es una Ley diseñada para ser desarrollada por las Comunidades en la que el Estado solamente fija el mínimo imprescindible en todo el territorio nacional. Corresponderá a cada territorio, en su caso, el incrementar ese mínimo. Debemos indicar que, a día de hoy, solo Andalucía y Cataluña han recogido el guante, publicando la Ley 11/2011, de 5 de diciembre, por la que se regula el uso de la lengua de signos española y los medios de apoyo a la comunicación oral de las personas sordas, con discapacidad auditiva y con sordoceguera en Andalucía; y la Ley 17/2010, de 3 de junio, de la lengua de signos catalana, respectivamente.

Si de los principios e ideas básicas de la Ley dependiera nuestra percepción sobre la misma, deberíamos estar más que satisfechos. En este sentido, la norma se fundamenta en los principios de: accesibilidad universal, transversalidad de las políticas en materia de lengua de signos, libertad de elección, no discriminación y, sobre todo, normalización; para construir un sistema de utilización libre en las siguientes áreas:

- $\quad$ Bienes y servicios a disposición del público

- Transportes

- Relaciones con las Administraciones Públicas

- Participación política

- Medios de comunicación social, telecomunicaciones y sociedad de la información

Pero nuestra percepción real es que la norma no baja al caso concreto y no establece un régimen real de protección y respeto a la Lengua de Signos española. Las proclamaciones generales de la norma no se acompañan de un régimen sancionador o infractor adecuado. Si bien se derivaban muchas cuestiones, como norma supletoria, a la hoy derogada Ley 51/2003 (de Igualdad de Oportunidades, No Discriminación y Accesibilidad Universal de las Personas con Discapacidad), lo cierto es que esta tampoco contenía, per se, un régimen sancionador que fuera más allá de ciertos mecanismos de solución extrajudicial de conflictos. Además, y pese a la Disposición Final Tercera (que obliga a destinar una partida presupuestaria al cumplimiento de la norma), los medios con los que, hoy día, cuentan los Servicios de Interpretación de las Federaciones de Personas Sordas son muy escasos.

En cuanto a la información se refiere, el artículo 14 se pronuncia en unos términos muy similares al Convenio de Nueva York:

- «1. Los poderes públicos garantizarán las medidas necesarias para que los medios de comunicación social, de conformidad con lo previsto en su regulación específica, sean accesibles a las personas sordas, con discapacidad auditiva y sordociegas mediante la incorporación de las lenguas de signos españolas.

- 2. Asimismo, los poderes públicos adoptarán las medidas necesarias para que las campañas de publicidad institucionales y los distintos soportes audiovisuales en los que éstas se pongan a disposición del público sean accesibles a estas personas.

- 3. Se establecerán las medidas necesarias para incentivar el acceso a las telecomunicaciones en lenguas de signos españolas.

- 4. Las páginas y portales de Internet de titularidad pública o financiados con fondos públicos se adaptarán a los estándares establecidos en cada momento por las autoridades competentes para lograr su accesibilidad a las personas sordas, con discapacidad auditiva y sordociegas mediante la puesta a disposición dentro de las mismas de los correspondientes sistemas de acceso a la información en la lengua correspondiente a su ámbito lingüístico.

- 5. Cuando las Administraciones Públicas promuevan o subvencionen Congresos, Jornadas, Simposios y Seminarios en los que participen personas sordas, con discapacidad auditiva y sordociegas, facilitarán su accesibilidad mediante la prestación de servicios de intérpretes en lengua de signos española y/o en las lenguas de signos propias de las Comunidades Autónomas si las hubiera, previa solicitud de los interesados.

- 6. Los mensajes relativos a la declaración de estados de alarma, excepción y sitio, así como los mensajes institucionales deberán ser plenamente accesibles a todas las personas sordas, con discapacidad auditiva y sordociegas»

Así, nos encontramos ante un régimen básico, con una dotación presupuestaria limitada (nótese que la Ley se publica en la época previa a la crisis económica, y quizá peca de un cierto optimismo en cuanto a los medios) y con un compromiso político que, a la vista del trabajo increíble de Asociaciones y Federaciones de Personas Sordas así como de la Confederación Nacional, se nos antoja como insuficiente. En materia de información, si bien sí se han producido avances, y se siguen produciendo, no podemos decir que se haya producido, ni mucho menos, la accesibilidad universal. Hoy no existe un acceso universal a bienes culturales 0 a información por parte de personas sordas 0 sordociegas. Un caso paradigmático ha sido la crisis del ébola. A diario veíamos informaciones sanitarias de naturalezas diversas que, desde luego, llegaban a personas oyentes. Las personas con discapacidad auditiva, por contra, recibieron información tardía y a cuentagotas, en un clima de preocupación y angustia en el que, desde luego lo aconsejable, habría sido una información accesible, por parte del Ministerio de Sanidad, desde el primer momento.

Apoya esta apreciación el Informe de seguimiento sobre España elaborado por el CERMI (Comité de Representantes de Personas con Discapacidad). En este documento, que analiza el cumplimiento por el Estado de las obligaciones del Convenio de Nueva York, denuncia, en las páginas 30 a 34, la falta de compromiso del Gobierno en el establecimiento de normas básicas en telecomunicaciones que garanticen el uso de la Lengua de Signos; al tiempo que señala la pasividad de las Comunidades Autónomas en el desarrollo legislativo de la norma básica estatal. Además, se hace mención de la falta de desarrollo reglamentario de la norma 51/2003 en materia de acceso a bienes y servicios públicos por personas con discapacidad. Antes de la derogación de la norma, el Reglamento se había retrasado ya más de 9 años, en una cuestión clave para el ejercicio del Derecho a la información. 


\subsection{El Texto Refundido de derechos de las personas con discapacidad}

En 2013 se aprueba en España el que es ahora el texto más importante en materia de los derechos de personas con discapacidad en nuestro país. Y es tal porque acumula y coordina tres normas que eran ya de vital importancia:

- La Ley 13/1982, de 7 de abril, de integración social de las personas con discapacidad.

- La Ley 51/2003, de 2 de diciembre, de igualdad de oportunidades, no discriminación y accesibilidad universal de las personas con discapacidad.

- La Ley 49/2007, de 26 de diciembre, por la que se establece el régimen de infracciones y sanciones en materia de igualdad de oportunidades, no discriminación y accesibilidad universal de las personas con discapacidad.

De este modo podemos observar, en una misma norma, el régimen sancionador completo por actividades discriminadoras en materia de discapacidad (artículo 1.b). Se trata, por tanto y como se indica en su objeto, de una norma tendente al ejercicio efectivo de los derechos, de su exigencia real. Así, al menos en el espíritu, es la norma que emplearemos para hacer positivos los derechos que hemos visto en el Convenio y en la Ley 27/2007. ¿Frente a qué protegerá la norma? Frente a discriminaciones, entendidas estas en un sentido amplio. Tomando de mano las definiciones de la propia Ley, protegerá contra:

- «Discriminación directa: es la situación en que se encuentra una persona con discapacidad cuando es tratada de manera menos favorable que otra en situación análoga por motivo de o por razón de su discapacidad.

- Discriminación indirecta: existe cuando una disposición legal o reglamentaria, una cláusula convencional o contractual, un pacto individual, una decisión unilateral o un criterio o práctica, o bien un entorno, producto o servicio, aparentemente neutros, puedan ocasionar una desventaja particular a una persona respecto de otras por motivo de o por razón de discapacidad, siempre que objetivamente no respondan a una finalidad legítima y que los medios para la consecución de esta finalidad no sean adecuados y necesarios.

- Discriminación por asociación: existe cuando una persona o grupo en que se integra es objeto de un trato discriminatorio debido a su relación con otra por motivo o por razón de discapacidad.

- Acoso: es toda conducta no deseada relacionada con la discapacidad de una persona, que tenga como objetivo o consecuencia atentar contra su dignidad o crear un entorno intimidatorio, hostil, degradante, humillante u ofensivo»

Como vemos, la norma establece, de momento, un régimen completo y cerrado contra toda actividad que perturbe la igualdad real de los individuos, con y sin discapacidad. Toda acción de diferenciación de trato, que tenga como razón última la discapacidad, y que sitúe a las personas con discapacidad en una posición de desventaja será sancionada como actividad discriminadora. Y este régimen alcanza también a los poderes públicos e incluso en su actividad legislativa. Para esto la Ley, como veíamos en las anteriores, se arma de una serie de principios inspiradores. En lo que a la información influye, destacaremos: libertad de toma de decisiones (informadas), vida independiente, no discriminación, igualdad de oportunidades, normalización, accesibilidad y diseño universales y participación social plena. Estos principios, y las normas del articulado, se aplicarán a los siguiente ámbitos materiales:

- Telecomunicaciones y sociedad de la información.

- Espacios públicos urbanizados, infraestructuras y edificación.

- Transportes.

- Bienes y servicios a disposición del público.

- Relaciones con las administraciones públicas.

- Administración de justicia.

- Patrimonio cultural, de conformidad con lo previsto en la legislación de patrimonio histórico.

- Empleo.

Por virtud del Texto Refundido, los Poderes Públicos asumen numerosas obligaciones. Se trata así de un cuerpo legal que les vincula y que puede serles exigidos. En lo que a la información se refiere estas obligaciones se ven reforzadas pues de este modo lo manifiesta el art. 7, indicando un deber reforzado de protección en «comunicación, información y acceso a la cultura». Puede demandarse también (art. 79) de personas jurídicas el cumplimiento de la Ley, a través de su régimen sancionador y sus mecanismos de solución de conflictos, pues en cuanto son prestadores de servicios al público, deben respetar la igualdad, y evitar discriminaciones.

Como hemos anunciado ya, el Texto Refundido se diferencia de otras normas en materia de discapacidad por contener varios sistemas de solución de conflictos y, además, un régimen sancionador completo. Este se aplicará a las situaciones ya vistas de discriminación directa, indirecta y actuaciones de acoso realizadas a personas con discapacidad, con independencia del reconocimiento oficial de tal, es decir, no es preciso poseer el certificado administrativo correspondiente para estar amparado por esta norma. Así, puede actuarse contra la discriminación de dos formas diferentes: con carácter previo y tras el acto en sí y/o a través de medidas de defensa. En cuanto a la primera categoría, la Ley prevé medidas de acción positiva (art. 67 y 68) y medidas de fomento y concienciación (art. 70 a 73 ).

En cuanto a los medios de defensa, la Ley se ocupa en primer lugar del arbitraje. A través de este medio el ofendido y el agente de discriminación pueden alcanzar un acuerdo, vinculante y con fuerza ejecutiva ante los Tribunales. Si bien el acceso a este medio se configura como voluntario, sí creemos que tiene ventajas interesantes, al ahorrar a las partes el desembolso de eventuales honorarios de abogado y procurador y, si se diese el caso, demás gastos de tramitación judicial.

En segundo lugar, la norma contiene también una serie de especificaciones en materia defensa judicial ante casos de discriminación. Podrá solicitarse, así, la cesación de los actos vulneradores de derechos, a la vez que solicitar medidas para que no vuelvan a repetirse. 
Además, por supuesto, podrá solicitarse del Tribunal la indemnización correspondiente que, en principio, no se encuentra limitada por tope máximo e incluirá daño moral. Por último, y como cuestión relevante, se incluye una suerte de inversión de la carga de la prueba por cuanto si la parte demandante presenta indicios de discriminación, será el demandado quien deberá probar la actuación conforme a Derecho. Se responde así a una reivindicación de las entidades de acción social pues en ocasiones resulta difícil o casi imposible probar que la razón de una diferencia de trato ha sido la propia discapacidad.

En tercer lugar, el régimen de reparación de daños o de satisfacción del individuo agraviado con un acto de discriminación se completa con un sistema de infracciones y sanciones. Estas, graduadas en leves, graves y muy graves, irán desde los $301 €$ hasta $1.000 .000 €$ en función de criterios como: intencionalidad del infractor, incumplimiento de advertencias previas, número de afectados y reincidencia. Además de esta sanción monetaria se prevé que pueda limitarse el acceso del infractor a subvenciones públicas o cualquier otro tipo de ayudas. El procedimiento para imponer estas sanciones, que deberá ser necesariamente accesible, podrá incluir la publicación de las sanciones.

Si bien parece un sistema atractivo sí debemos hacer una labor de crítica. En este sentido, solamente se encuentra vigente para la Administración General del Estado, requiriendo para su aplicación a Comunidades Autónomas que estas desarrollen las previsiones del Texto Refundido. Teniendo en cuenta la competencia autonómica en Servicios Sociales, Sanidad, Educación y muchas otras, resulta muy limitativo un sistema en el que no puede exigírseles, en virtud de esta norma y de momento, el acceso universal a información. Además, y en vista de los escasos esfuerzos de las Administraciones Públicas en el cumplimiento de sus obligaciones en materia de discapacidad, no podemos tener confianza plena en que la adaptación reglamentaria autonómica se produzca en un tiempo razonable.

\section{Cómo citar este artículo en bibliografías - How to cite this article in bibliographies / references:}

VIEITO-VILLAR, M. (2015): "La discriminación en el acceso a la información en la legislación española de discapacidad". En Revista de la Asociación Española de Investigación de la Comunicación, 2, pp. 64-70. 